\title{
CASE REPORT: COMPLICATION AT BOTH RECEIVING AND DONOR SITES IN FACIAL AUTOLOGOUS FAT TRANSFER
}

\author{
Vu Ngoc Lam*, Le Thi Thu Hai*, Nguyen Phuong Tien*
}

\section{ABSTRACT}

Autologous fat grafting is a technique that has been used for a long time in the field of plastic surgery. Besides many advantages, the complications, which can range from mild such as hematoma, infection, calcification, necrosis... even death is a big problem. The article presents a case of using autologous fat taken from the abdomen to graft onto the facial area with complications of severe infection in both the receiving and donor sites, causing necrosis of fat and skin in both areas. The treatment takes a long time with different procedures, but the consequences for the patient are still very serious both in terms of aesthetics and function. The objective of this study was to evaluate the safety and efficacy of fat grafting to the facial region for the reconstruction and aesthetic enhancement of facial contour.

Keywords: Autologous fat graft; Facial region; Complications

\section{INTRODUCTION}

The first author to describe the transfer of fat as a means of treating retractile scarring in 1893. Fat grafting, however, has only gained widespread popularity amongst plastic surgeons over the past several decades. Its use as a treatment for both cosmetic and reconstructive contour defects has evolved due to the development of modern liposuction in the 1980's by Illouz

\footnotetext{
* 108 Military Central Hospital

Responsible person: Vu Ngoc Lam

Email: vungoclamb8@gmail.com

Date of receipt: 03/6/2021

Date of scientific judgment: 07/7/2021

Reviewed date: 17/8/2021
}

and the continued refinement of harvesting, preparation and grafting techniques such as those pioneered by Coleman.

Fat serves as ideal soft tissue filler in that it is autologous, biocompatible, easily accessible in most patients, relatively permanent and can integrate into the surrounding tissue at the injection site. Its use in the facial region has also increased, although less is published regarding its safety and effectiveness for facial reconstruction or aesthetic improvement.

In Vietnam, autologous fat injection become more and more popular and has brought many of excellent results. But in the other corner, the abuse of this technique especially by untrained practitioners would cause many terrible complications. We would like to present a clinical case which suffered from serious infection and skin necrosis in both receiving and donor areas.

\section{CASE REPORT}

A 53-year-old lady has been injected bout $40 \mathrm{cc}$ of autologous fat which is taken from the abdomen to the cheeks and chin areas for the purpose of improving the shape of the face. The procedure was smooth, the patient was given oral antibiotics and antiinflammatory for 5 days.

2 weeks after fat injection, the patient found that her face was swollen, her cheeks were erythema and painful. The fat donor area in the abdomen is also swollen, red, and painful. 

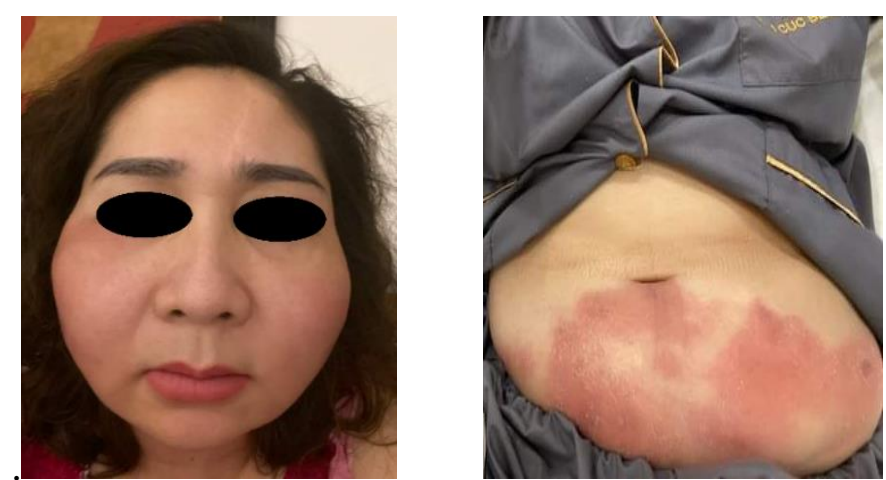

Pictures 1-2: The erythema swollen cheek and abdominal 2 weeks after fat transfer

The patient continued to use antibiotics but did not see improvement and was admitted to a local hospital for treatment. The doctor at that hospital used high-dose
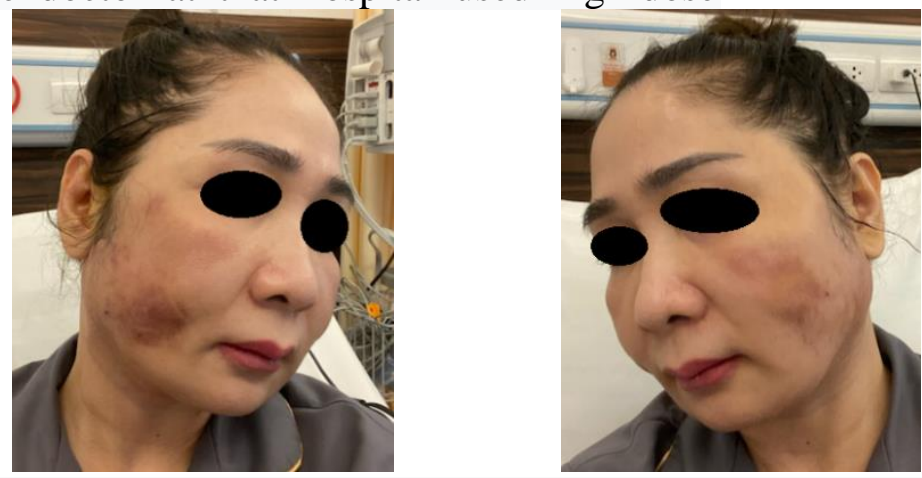

strong antibiotics, made incisions to remove pus from the cheeks and abdomen, and drainages were placed in holes.

Pictures 3-4-5: The infection focis in both face and abdominal after first intervention

After 2 weeks of treatment, the infection still did not improve, the patient was transferred to 108 Central Military Hospital (108 CMH) for treatment.

Examination at hospital admission 108 $\mathrm{CMH}$ : The patient has no fever but felt very tired. The cheeks, submandibular area, anterior - inferior of ears, and the abdomen below the umbilicus have many red, inflamed, purulent lesions.

Collecting pus fluid to test bacteria, fungi, make antibiotic chart: the results showed no fungus, but the presence of myobacterium.

The patient was supplemented with intravenous nutrition, surgically injecting pus, cleaning, and removing necrotic tissue.
After the necrotic foci were cleaned and healed, after about 5-7 days, new inflammatory foci of similar characteristic appeared at new sites (these sites were not injected with fat before).

In the facial area, there were about 4 times the formation of new inflammatory-necrotic foci. In the abdominal region, the number of new necrotic foci of formation is less, but the foci are interconnected like a subcutaneous tunnel system.

We removed the entire skin-fat containing necrotic foci in the abdomen and sutured directly. After surgery, the wound healed peacefully in 10 days. Total time for the treatment is 3 months. 

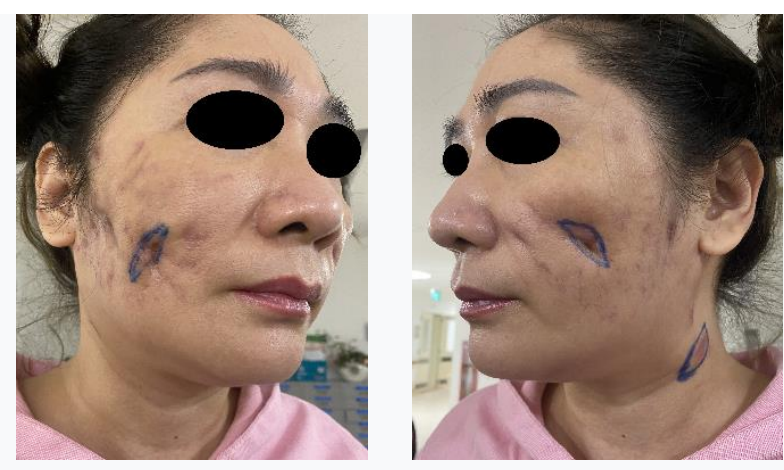

Pictures 6-7: Poly necrosis focies excisions on the facial area
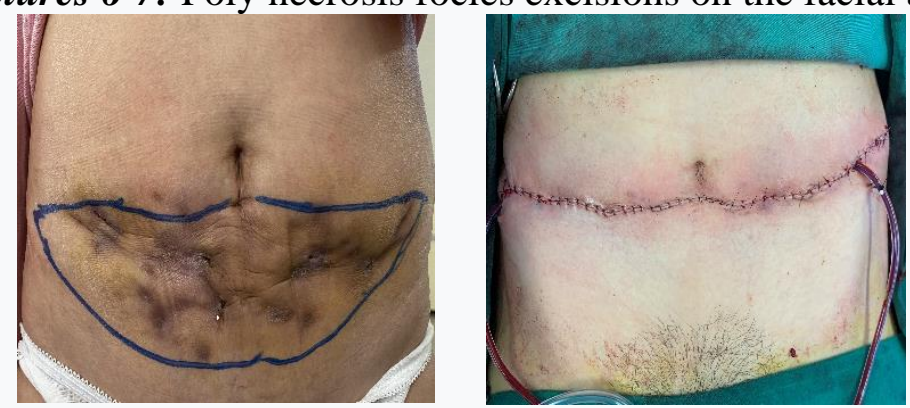

Pictures 8-9: The total piece of necrosis area in abdominal was cut and primary close
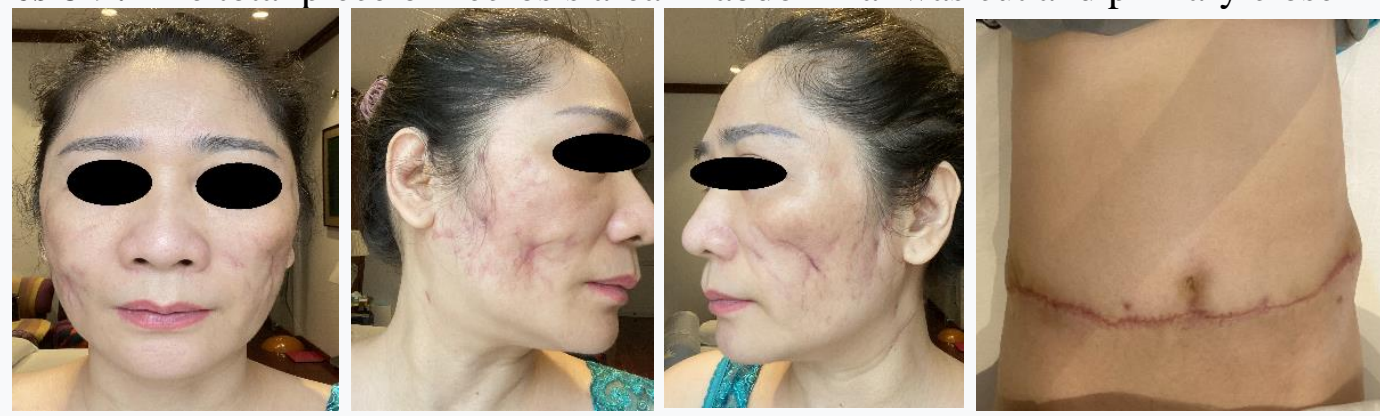

Pictures 10-11-12-13: Scars on face and abdominal 1 month later

\section{DISCUSSIONS}

Autologous fat transferring has many beneficial qualities that make it advantageous for correcting contour deformities or augmenting soft-tissue for reconstructive or cosmetic indications. Fat grafts are autologous, biocompatible, permanent and viable when integrated into the injection site. They are easily accessible in most patients, and represent a relatively simple and lowcost surgical procedure [1].

Our patient decided to choose autologous fat injection for facial contouring because she was consulted that is a very safe and effective method, easy to apply and giving a very quick natural result.

The results of many studies serve to expand the existing body of literature and provide more complete evidence, pooled from the existing data, describing the effectiveness and safety of autologous fat grafting for the treatment of facial contour defects. But they also illustrate the versatility of fat grafting, while also highlighting important details such as complication rates and rates of graft retention relative to anatomic regions of the face and processing techniques[7]. 
The complication rate $(2.27 \%)$ identified was minimal, with the majority of complications $(84.61 \%)$ being attributed to either asymmetry or skin irregularities (excessive or inadequate grafting or graft survival). Fat necrosis $(n=3)$ and infection $(n=2)$ represented $2.88 \%$ and $1.92 \%$ of all complications, respectively. These results differ from those seen in fat grafting used for both breast and gluteal augmentation. In the systematic review by Voglimacci et al., the authors reported a complication rate of $12.6 \%$ in cosmetic breast fat grafting. Of the 256 documented complications, there were 60 cases of fat necrosis $(23.44 \%$ of all complications), and 18 cases of infection (7.03\% of all complications). Conde-Green et al. reported an overall complication rate of $7 \%$ in gluteal fat augmentation, of which there were 24 cases of infection $(8.33 \%$ of all complications), and 23 cases of fat necrosis (7.99\% of all complications) [2]. Complications were erythema, prolonged edema (lasting greater than 3 weeks), telangiectasia, asymmetry, skin irregularities, graft hypertrophy, erythema, infection and fat necrosis.

In this case, the infection and fat necrosis happened in both receiving and donor sites so we consider the cause of this complication strongly related to the instruments used in during the fat collection and fat injection.

Certain studies have therefore suggested using blunt cannulas to minimize this risk during injection in addition to aspiration prior to injection. Unfortunately, there was insufficient data in the analyzed papers to validate this claim. In the present review, there were no reports of mortality or significant embolic complications associated with facial fat grafting procedures [3].
Our case demonstrates a rare though possible complication of autologous fat transfer. Fat necrosis of the newborn is recognized as an entity in literature and fat necrosis post autologous fat transfer to the breast has also been described. Fat necrosis resulting from autogenous fat transfer performed for facial filling has however been less extensively reported and is noteworthy of recognition especially due to the potential of permanent scarring, normally the infection and necrosis happened in the receiving area but in this patient that occured in both sites.

There may be several factors relevant to the causation of fat necrosis post transfer which include preoperative patient selection, intra operative and post operative risk factors. Special consideration must be given to the identification of any underlying inflammatory dermatoses prior to the performance of autogenous fat transfer and ensuring correct patient selection for this procedure. An underlying active inflammatory dermatosis is likely to accelerate lipolysis of the transferred fat due to the body's inherent defence response to areas of infection or inflammation [5].

Autologous fat transfer as a procedure requires operator skill as well as practise and precision. This sets this filling procedure apart from other intradermal fillers as a high level of skill is involved in the harvesting, the preparation and the replacement of the fat. Factors that have been reported to improve graft survival include:

1. Low vascularity of the donor site.

2. High vascularity of the recipient site.

3. A low-pressure technique used to aspirate the fat.

4. Skillful washing and preparation of fat.

5. A sufficiently large cannula. 
6. The use of a multilayered approach to replace the fat.

Autologous fat transfer may lead to complications other than fat necrosis. The early recognition and treatment of these enhances a clinician's procedure prowess [4].

Some other complications include:

Rapid absorption of the replaced fat. This is exacerbated by the presence of blood; damaged fat cells and high-pressure technique.

Infection presenting pre, intra or post operatively may affect graft survival. Viral infections or warty growths have also been reported at the site of cannula entry [6].

Skin necrosis and sinus formation may result from overfilling or over augmentation of the desired site.

This case of fat necrosis was treated with serial staged excisions of the affected areas by applying basic principles of dermatologic surgery to the treatment of an unusual cause of scarring.

\section{CONCLUSIONS}

Autologous fat grafting is not a simple procedure and should be performed by welltrained and skilled surgeons. Patients should be informed that it is associated with a risk of some side effects. Fat and skin necrosis in both recipient and donor sites in facial autologous fat grafting is a rare complication. The main reason for this serious complication is the used of sub-stands sterilized instruments during the technical procedure. The treatment including using high-dose antibiotics, anti-inflammatory drugs, strengthening the body's resistance and surgical measures are decisive. Need multiple surgeries to remove pus, debris necrosis and infiltrated tissue. The treatment process is often lengthy and leaves serious consequences both aesthetically and functionally for the patient.

\section{REFERENCES}

1. S.R. Coleman. Facial recontouring with lipostructure. Clin Plast Surg, 24 (1997), pp. 347-367

2. M. Voglimacci, I. Garrido, A. Mojallal, et al. Autologous fat grafting for cosmetic breast augmentation: a systematic review. Aesthet Surg J, 35 (4) (2015), pp. 378-393

3. Yu N.Z., Huang J.Z., Zhang H., et al. A systemic review of autologous fat grafting survival rate and related severe complications. Chinese Med J, 128 (9) (2015), pp. 1245-1251

4. S.L. Spear, C.N. Coles, Leung B.K., M. Gitl in, M. Parekh, D. Macarios. The safety, effectiveness, and efficiency of autologous fat grafting in breast surgery. Plast Reconstruct Surg Global Open, 4 (8) (2016), p. e827

5. A. Mojallal, C. Shipkov, F. Braye, P. Breto n, J.L. Foyatier. Influence of the recipient site on the outcomes of fat grafting in facial reconstructive surgery. Plast Reconstr Surg, 124 (2) (2009), pp. 471-483

6. Xie Y., Li Q., Zheng D. Treatment of autologous fat injection for hemifacial atrophy. Chinese J Repar Reconstruct Surg, 21 (12) (2007), pp. 1308-1311

7. M.E. Ramil. Fat grafting in hollow upper eyelids and volumetric upper blepharoplasty. Plast Reconstr Surg, 140 (5) (2017), pp. 889897 